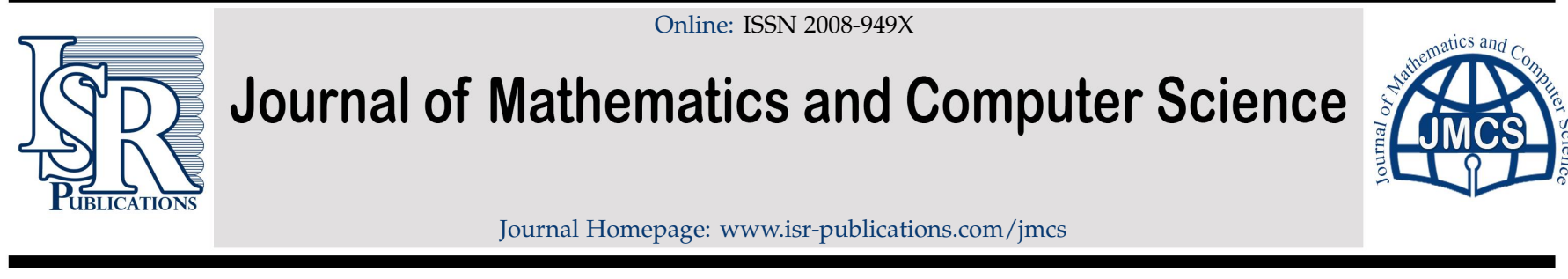

\title{
Null boundary controllability of nonlinear integrodifferen- tial systems with Rosenblatt process
}

\author{
Mahmoud A. AL-Nahhas ${ }^{a}$, Hamdy M. Ahmed ${ }^{b, *}$, Hassan M. El-Owaidyc \\ a International Academy for Engineering and Media Sciences, Cairo, Egypt. \\ ${ }^{b}$ Higher Institute of Engineering, El-Shorouk Academy, El-Shorouk City, Cairo, Egypt. \\ ${ }^{c}$ Faculty of Sciences, Al-Azhar University, Cairo, Egypt.
}

\begin{abstract}
By using stochastic analysis, compact semigroups and Schauder fixed-point theorem, we discuss the null boundary controllability of nonlinear integrodifferential system with Rosenblatt process. In addition, the null boundary controllability of Sobolev-type neutral integro-differential system with Rosenblatt is studied. Finally, an example is given to illustrate the obtained results. The null controllability results for stochastic differential systems with Rosenblatt process and control on the boundary have not yet been considered in the literature, and this fact motivates this work.
\end{abstract}

Keywords: Rosenblatt process, stochastic analysis, Sobolev-type integro-differential system, null boundary controllability.

2020 MSC: 93B05, 93C10, 34A08, 60G22.

(C)2022 All rights reserved.

\section{Introduction}

In recent years fractional differential equations have attracted the attentions of many researchers and are becoming increasingly popular due to their practical applications in various fields of science and engineering (see $[1,9,17,26,30,37])$. The deterministic system often fluctuates due to environmental noise. So, it is important and necessary for us to deal with stochastic differential equations (SDEs). The noises arise in mathematical finance, physics, telecommunication networks, hydrology and medicine etc., can be modeled by fractional Brownian motions ( $\mathrm{fBm})$. The $\mathrm{fBm}$ can be expressed as a Wiener integral with respect to the standard Wiener process, i.e. the integral of a deterministic kernal with respect to a standard Brownian motion, the Hermite process of order 1 is $\mathrm{fBm}$ and of order 2 is the Rosenblatt process. Firstly, Tudor investigated the Rosenblatt process which is a self-similar process with stationary increments and it appears as limit of long-range dependent stationary series in the Non-Central Limit theorem, for more details see $[2,7,29,35]$. Stochastic differential equations driven by fractional Brownian motion have been considered greatly by research community in various aspects due to its salient features for real world problems (see $[8,15,16,20,31,34]$ ). On the other hand, controllability problems for

\footnotetext{
*Corresponding author

Email addresses: amro_alx@hotmail.com (Mahmoud A. AL-Nahhas), hamdy_17eg@yahoo.com (Hamdy M. Ahmed), elowaidy@yahoo.com (Hassan M. El-Owaidy)
}

doi: $10.22436 /$ jmcs.026.02.02

Received: 2020-10-23 Revised: 2021-03-10 Accepted: 2021-08-13 
different kinds of dynamical systems have been studied by several authors (see [3, 5, 10, 12, 18, 19, $21,32,36]$, and references therein). There are many applications for boundary control systems (see $[22,24])$. Many authors studied the controllability for linear and nonlinear system when the control on the boundary, for example, Barbu studied the boundary control problems with convex cost criterion (see [14]). Balachandran and Anandhi discussed the boundary controllability for ntegrodifferential systems and delay integrodifferential systems in Banach spaces (see [11, 13]). Ahmed obtained the boundary controllability of nonlinear fractional integrodifferential systems (see [4]). Lizzy and Balachandran studied the boundary controllability of nonlinear stochastic fractional system in Hilbert space (see [25]). Ahmed studied the boundary controllability of impulsive nonlinear fractional delay integrodifferential system (see [6]). Wang established sufficient condition for approximate boundary controllability for Semilinear delay differential equations (see [36]). Palanisamy and Chinnathambi investigated the approximate boundary controllability of Sobolev-type stochastic differential systems in Hilbert spaces (see [27]). However, the null controllability results for stochastic differential systems with Rosenblatt process and control on the boundary have not yet been considered in the literature, and this fact motivates this work.

The contributions of this paper exist in the following aspects.

- Stochastic differential systems with Rosenblatt process and control on the boundary are introduced.

- Sufficient conditions for null boundary controllability of nonlinear integrodifferential system with Rosenblatt process are established.

- Sufficient conditions for null boundary controllability of Sobolev type neutral stochastic integrodifferential system with Rosenblatt process are established.

This paper is prepared as follows. In Section 2, we present some basic definitions and lemmas which are useful to prove the main results. In Section 3, we give sufficient conditions to prove the null boundary controllability of nonlinear integrodifferential system with Rosenblatt process. In Section 4, we study the null boundary controllability of Sobolev-type neutral integrodifferential system with Rosenblatt process. In Section 5, we consider an example to verify the theoretical results.

\section{Preliminaries}

Let $(\Omega, F, P)$ be a complete probability space equipped with a normal filtration $F_{t}, t \in[0, b]$ where $F_{t}$ is the $\sigma$-algebra generated by random variables $\left\{\omega(s), Z_{H}(s), s \in[0, b]\right\}$ and all P-null sets.

Fix a time interval $[0, T]$ and let $\left\{Z_{H}(t), t \in[0, T]\right\}$ represents one-dimensional Rosenblatt process with parameter $H \in(1 / 2,1)$. The Rosenblatt process with parameter $H>\frac{1}{2}$ can be written as [28]

$$
Z_{H}(t)=d(H) \int_{0}^{t} \int_{0}^{t}\left[\int_{y_{1} \vee y_{2}}^{t} \frac{\partial K^{H^{\prime}}}{\partial v}\left(v, y_{1}\right) \frac{\partial K^{H^{\prime}}}{\partial v}\left(v, y_{2}\right)\right] d B\left(y_{1}\right) d B\left(y_{2}\right),
$$

where $\mathrm{K}^{\mathrm{H}}(\mathrm{t}, \mathrm{s})$ is given by

$$
\mathrm{K}_{\mathrm{H}}(\mathrm{t}, \mathrm{s})=\mathrm{c}_{\mathrm{H}} \mathrm{s}^{\frac{1}{2}-\mathrm{H}} \int_{\mathrm{s}}^{\mathrm{t}}(v-\mathrm{s})^{\mathrm{H}-\frac{3}{2}} v^{\mathrm{H}-\frac{1}{2}} \mathrm{~d} v, \text { for } \mathrm{s}<\mathrm{t},
$$

with $c_{H}=\sqrt{\frac{H(2 H-1)}{\beta\left(2-2 \mathrm{H}, \mathrm{H}-\frac{1}{2}\right)}}$ and $\beta(\cdot, \cdot)$ denotes the Beta function, $\mathrm{K}_{\mathrm{H}}(\mathrm{t}, \mathrm{s})=0$ when $\mathrm{t} \leqslant \mathrm{s},\{\mathrm{B}(\mathrm{t}), \mathrm{t} \in[0, \mathrm{~T}]\}$ is a Brownian motion, $\mathrm{H}^{\prime}=\frac{\mathrm{H}+1}{2}$ and $\mathrm{d}(\mathrm{H})=\frac{1}{\mathrm{H}+1} \sqrt{\frac{\mathrm{H}}{2 \mathrm{H}-1}}$ is a normalizing constant. The covariance of the Rosenblatt process $Z_{H}(t), t \in[0, T]$ satisfies $E\left(Z_{H}(t) Z_{H}(s)\right)=\frac{1}{2}\left(s^{2 H}+t^{2 H}-|s-t|^{2 H}\right)$.

Let $X$ and $Y$ be two real, separable Hilbert spaces and let $L(Y, X)$ be the space of bounded linear operators from $Y$ to $X$. For the sake of convenience, we shall use the same notation to denote the norms in $X, Y$ and $L(Y, X)$. Let $Q \in L(Y, Y)$ be an operator defined by $Q e_{n}=\lambda_{n} e_{n}$ with finite trace $\operatorname{trQ}=$ 
$\sum_{n=1}^{\infty} \lambda_{n}<\infty$ where $\lambda_{n} \geqslant 0(n=1,2, \ldots)$ are non-negative real numbers and $\left\{e_{n}\right\}(n=1,2, \ldots)$ is a complete orthonormal basis in $\mathrm{Y}$. We define the infinite dimensional Q-Rosenblatt process on $\mathrm{Y}$ as

$$
\mathrm{Z}_{\mathrm{H}}(\mathrm{t})=\mathrm{Z}_{\mathrm{Q}}(\mathrm{t})=\sum_{\mathrm{n}=1}^{\infty} \sqrt{\lambda_{\mathrm{n}}} e_{\mathrm{n}} z_{\mathrm{n}}(\mathrm{t}),
$$

where $\left(z_{\mathfrak{n}}\right)_{\mathfrak{n} \geqslant 0}$ is a family of real, independent Rosenblatt process.

In order to define Wiener integrals with respect to the Q-Rosenblatt process, we introduce the space $\mathrm{L}_{2}^{0}:=\mathrm{L}_{2}^{0}(\mathrm{Y}, \mathrm{X})$ of all Q-Hilbert Schmidt operators $\psi: \mathrm{Y} \rightarrow \mathrm{X}$. We recall that $\psi \in \mathrm{L}(\mathrm{Y}, \mathrm{X})$ is called a Q-Hilbert-Schmidt operator, if

$$
\|\psi\|_{\mathrm{L}_{2}^{0}}^{2}:=\sum_{n=1}^{\infty}\left\|\bar{\lambda}_{n} \psi e_{n}\right\|^{2}<\infty
$$

and that the space $L_{2}^{0}$ equipped with the inner product $\langle\vartheta, \psi\rangle_{L_{2}^{0}}=\sum_{n=1}^{\infty}\left\langle\vartheta e_{n}, \psi e_{n}\right\rangle$ is a separable Hilbert space. Let $\phi(s) ; s \in[0, T]$ be a function with values in $L_{2}^{0}(Y, X)$, the Wiener integral of $\phi$ with respect to $\mathrm{Z}_{\mathrm{Q}}$ is defined by

$$
\int_{0}^{t} \phi(s) d Z_{Q}(s)=\sum_{n=1}^{\infty} \int_{0}^{t} \sqrt{\lambda} K_{H}^{*}\left(\phi e_{n}\right)\left(y_{1}, y_{2}\right) d B\left(y_{1}\right) d B\left(y_{2}\right)
$$

where

$$
K_{H}^{*}(\vartheta)\left(y_{1}, y_{2}\right)=\int_{y_{1} \vee y_{2}}^{T} \vartheta(r) \frac{\partial K}{\partial r}\left(r, y_{1}, y_{2}\right) d r
$$

For more details (see [33]).

Lemma 2.1 ([23]). If $\psi:[0, \mathrm{~T}] \rightarrow \mathrm{L}_{2}^{0}(\mathrm{Y}, \mathrm{X})$ satisfies $\int_{0}^{\mathrm{T}}\|\psi(\mathrm{s})\|_{\mathrm{L}_{2}^{0}}^{2}<\infty$, then the above sum in (2.1) is well defined as $\mathrm{X}$-valued random variable and we have

$$
\mathrm{E}\left\|\int_{0}^{\mathrm{t}} \psi(\mathrm{s}) \mathrm{dZ} \mathrm{H}_{\mathrm{H}}(\mathrm{s})\right\|^{2} \leqslant 2 \mathrm{Ht}^{2 \mathrm{H}-1} \int_{0}^{\mathrm{t}}\|\psi(\mathrm{s})\|_{\mathrm{L}_{2}^{0}}^{2} \mathrm{ds} .
$$

The collection of all strongly-measurable, square-integrable, $\mathrm{X}$-valued random variables, denoted by $\mathrm{L}_{2}(\Omega, X)$, is a Banach space equipped with norm

$$
\|x(\cdot)\|_{\mathrm{L}_{2}(\Omega, X)}=\left(E\|x(., \omega)\|^{2}\right)^{\frac{1}{2}},
$$

where the expectation, $E$ is defined by $E(x)=\int_{\Omega} x(\omega) d P$. Let $C\left(J, L_{2}(\Omega, X)\right)$ be the Banach space of all continuous maps from $J$ into $L_{2}(\Omega, X)$ satisfying the condition $\sup _{t \in J} E\|x(t)\|^{2}<\infty$. Define $\bar{C}=\{x$ : $\left.x(t) \in \mathrm{C}\left(J, \mathrm{~L}_{2}(\Omega, X)\right)\right\}$, with norm $\|\cdot\|_{\overline{\mathrm{C}}}$ defined by

$$
\|\cdot\|_{\bar{C}}=\left(\sup _{t \in J} E\|x(t)\|^{2}\right)^{\frac{1}{2}} .
$$

Obviously, $\bar{C}$ is a Banach space. Also, let us introduce the set $B_{r}=\left\{v \in \bar{C}:\|v\|_{\overline{\mathrm{C}}}^{2} \leqslant r\right\}$, where $r>0$.

\section{Nonlinear integrodifferential system}

In this section, we study the null boundary controllability of nonlinear integrodifferential system with Rosenblatt process in the following form

$$
\begin{cases}\frac{d x(t)}{d t}=\sigma x(t)+f_{1}(t, x(t))+\int_{0}^{t} f_{2}(s, x(s)) d \omega(s)+g(t, x(t)) \frac{d Z_{H}(t)}{d t}, & t \in J=[0, b], \\ \tau x(t)=B_{1} u(t), & t \in J=[0, b] \\ x(0)=x_{0}, & \end{cases}
$$


where the state $x(\cdot)$ takes values in the separable Hilbert space $X$ with inner product $\langle.,$.$\rangle and norm \|$. and the control function $u(\cdot)$ is given in $\mathrm{L}_{2}(\mathrm{~J}, \mathrm{U})$, the Hilbert space of admissible control functions with $\mathrm{U}$ a Hilbert space. Here, $\sigma, \mathrm{B}_{1}$ are bounded linear operators and $\tau$ is a linear operator with domain in $X$ and range in separable Hilbert space $Y$. Let $A: X \rightarrow X$ be the linear operator defined by $D(A)=\{x \in$ $\mathrm{D}(\sigma) ; \tau x=0\}, A x=\{\sigma x$, for $x \in D(A)\}$. Suppose $\{\omega(t)\}_{t \geqslant 0}$ is a Wiener process defined on $\left(\Omega, F,\left\{F_{t}\right\}_{t} \geqslant 0, P\right)$ with values in Hilbert space $K$ and $\left\{Z_{H}(t)\right\}_{t} \geqslant 0$ is a Rosenblatt process with parameter $H \in\left(\frac{1}{2}, 1\right)$ defined on $\left(\Omega, F,\left\{F_{t}\right\}_{t \geqslant 0}, P\right)$ with values in Hilbert space $Y$. The nonlinear operators $f_{1}: J \times X \rightarrow X, f_{2}: J \times X \rightarrow L(K, X)$ and $\mathrm{g}: \mathrm{J} \times \mathrm{X} \rightarrow \mathrm{L}_{2}^{0}(\mathrm{Y}, \mathrm{X})$ are given. To establish the result, we need the following hypotheses.

(H1) $\mathrm{D}(\sigma) \subset \mathrm{D}(\tau)$ and the restriction of $\tau$ to $\mathrm{D}(\sigma)$ is continuous relative to graph norm of $\mathrm{D}(\sigma)$.

(H2) There exists a linear continuous operator $B: U \rightarrow X$ such that $\sigma B \in L(U, X) ; \tau(B u)=B_{1} u$ for all $u \in U$. Also $B u(t)$ is continuously differentiable and $\|B u\| \leqslant C\left\|B_{1} u\right\|$ for all $u \in U$, where $C$ is some positive constant.

(H3) The operator $A$ is the infinitesimal generator of a compact semigroup $T(t)$ on $X$ and there exists a constant $M>0$ such that $\|T(t)\| \leqslant M$.

(H4) For all $t \in(0, b]$ and $u \in U, T(t) B u \in D(A)$. Moreover, there exists a positive constant $M_{1}>0$ such that $\|A T(t)\| \leqslant M_{1}$.

(H5) The function $\mathrm{f}_{1}: \mathrm{J} \times \mathrm{X} \rightarrow \mathrm{X}$ satisfies the following two conditions:

(i) the function $f_{1}: J \times X \rightarrow X$ is continuous;

(ii) for each positive number $r \in N$, there is a positive function $\rho_{r}(\cdot): J \rightarrow R^{+}$such that

$$
\sup _{\|x\|^{2} \leqslant r} E\left\|f_{1}(t, x(t))\right\|^{2} \leqslant \rho_{r}(t),
$$

the function $s \rightarrow \rho_{r}(s) \in \mathrm{L}^{1}\left([0, \mathrm{t}], \mathrm{R}^{+}\right)$, and there exists a $\delta>0$ such that

$$
\lim _{r \rightarrow \infty} \inf \frac{\int_{0}^{t} \rho_{r}(s) d s}{r}=\delta<\infty, t \in J .
$$

(H6) The function $f_{2}: J \times X \rightarrow L(K, X)$ satisfies the following two conditions:

(i) the function $f_{2}: J \times X \rightarrow L(K, X)$ is continuous;

(ii) for each positive number $r \in N$, there is a positive function $h_{r}(\cdot): J \rightarrow R^{+}$such that

$$
\sup _{\|x\|^{2} \leqslant r} \int_{0}^{t} E\left\|f_{2}(s, x(s))\right\|_{Q}^{2} d s \leqslant h_{r}(t),
$$

the function $s \rightarrow h_{r}(s) \in L^{1}\left([0, t], R^{+}\right)$, and there exists a $\delta_{1}>0$ such that

$$
\lim _{r \rightarrow \infty} \inf \frac{\int_{0}^{t} h_{r}(s) d s}{r}=\delta_{1}<\infty, t \in J .
$$

(H7) The function $\mathrm{g}: \mathrm{J} \times \mathrm{X} \rightarrow \mathrm{L}_{2}^{0}(\mathrm{Y}, \mathrm{X})$ satisfies the following two conditions:

(i) the function $\mathrm{g}: \mathrm{J} \times \mathrm{X} \rightarrow \mathrm{L}_{2}^{0}(\mathrm{Y}, \mathrm{X})$ is continuous;

(ii) for each positive number $r \in N$, there is a positive function $k_{r}(\cdot): J \rightarrow R^{+}$such that

$$
\sup _{\|x\|^{2} \leqslant r} E\|g(t, x(t))\|_{L_{2}^{0}}^{2} \leqslant k_{r}(t)
$$

the function $s \rightarrow k_{r}(s) \in L^{1}\left([0, t], R^{+}\right)$, and there exists a $\delta_{2}>0$ such that

$$
\lim _{r \rightarrow \infty} \inf \frac{\int_{0}^{t} k_{r}(s) d s}{r}=\delta_{2}<\infty, t \in J .
$$


Let $x(t)$ be the solution of the system (3.1). Then we can define a function $z(t)=x(t)-B u(t)$ and from our assumption we see that $z(t) \in D(A)$. Hence (3.1) can be written in terms of $A$ and $B$ as

$$
\left\{\begin{array}{l}
\frac{d x(t)}{d t}=A z(t)+\sigma B u(t)+f_{1}(t, x(t))+\int_{0}^{t} f_{2}(s, x(s)) d w(s)+g(t, x(t)) \frac{d Z_{H}(t)}{d t}, t \in J, \\
x(t)=z(t)+B u(t), \\
x(0)=x_{0} .
\end{array}\right.
$$

If $u$ is continuously differentiable on $[0, b]$, then $z$ can be defined as a mild solution to the Cauchy problem

$$
\left\{\begin{array}{l}
\frac{d z(t)}{d t}=A z(t)+\sigma B u(t)-B \frac{d u(t)}{d t}+f_{1}(t, x(t))+\int_{0}^{t} f_{2}(s, x(s)) d \omega(s)+g(t, x(t)) \frac{d Z_{H}(t)}{d t}, t \in J \\
z(0)=x(0)-B u(0)
\end{array}\right.
$$

Then the mild solution of (3.2) is given by

$$
\begin{aligned}
z(t)= & T(t) z(0)+\int_{0}^{t} T(t-s)\left[\sigma B u(s)-B \frac{d u(s)}{d s}\right] d s+\int_{0}^{t} T(t-s) f_{1}(s, x(s)) d s \\
& +\int_{0}^{t} \int_{0}^{s} T(t-s) f_{2}(\eta, x(\eta)) d \omega(\eta) d s+\int_{0}^{t} T(t-s) g(s, x(s)) d Z_{H}(s) .
\end{aligned}
$$

Since, $x(t)=z(t)+B u(t)$, then the solution of (3.1) is given by

$$
\begin{aligned}
x(t)= & T(t)\left[x_{0}-B u(0)\right]+B u(t)+\int_{0}^{t} T(t-s)\left[\sigma B u(s)-B \frac{d u(s)}{d s}\right] d s \\
& +\int_{0}^{t} T(t-s) f_{1}(s, x(s)) d s+\int_{0}^{t} \int_{0}^{s} T(t-s) f_{2}(\eta, x(\eta)) d \omega(\eta) d s+\int_{0}^{t} T(t-s) g(s, x(s)) d Z_{H}(s) .
\end{aligned}
$$

By using integration by parts, we get

$$
\int_{0}^{t}\left[T(t-s) B \frac{d u(s)}{d s}\right] d s=B u(t)-T(t) B u(0)+\int_{0}^{t} A T(t-s) B u(s) d s .
$$

By substitution in (3.3) we obtain

$$
\begin{aligned}
x(t)= & T(t) x_{0}+\int_{0}^{t}[T(t-s) \sigma-A T(t-s)] B u(s) d s+\int_{0}^{t} T(t-s) f_{1}(s, x(s)) d s \\
& +\int_{0}^{t} \int_{0}^{s} T(t-s) f_{2}(\eta, x(\eta)) d \omega(\eta) d s+\int_{0}^{t} T(t-s) g(s, x(s)) d Z_{H}(s) .
\end{aligned}
$$

which it is a mild solution of (3.1). To study the exact null controllability of (3.1), we consider the linear boundary control system

$$
\begin{cases}\frac{d \mu(t)}{d t}=\sigma \mu(t)+F(t), & t \in J=[0, b] \\ \tau \mu(t)=B_{1} u(t), & t \in J=[0, b] \\ \mu(0)=\mu_{0}, & \end{cases}
$$

associated with the system (3.1). Consider

$$
\mathrm{L}_{0}^{\mathrm{b}} \mathrm{u}=\int_{0}^{\mathrm{b}}[\mathrm{T}(\mathrm{b}-\mathrm{s}) \sigma \mathrm{B}-\mathrm{AT}(\mathrm{b}-\mathrm{s}) \mathrm{B}] \mathrm{u}(\mathrm{s}) \mathrm{ds}: \mathrm{L}_{2}(\mathrm{~J}, \mathrm{U}) \rightarrow \mathrm{X},
$$

where $L_{0}^{b} u$ has a bounded inverse operator $\left(L_{0}\right)^{-1}$ with values in $L_{2}(J, u) / \operatorname{ker}\left(L_{0}^{b}\right)$, and

$$
\mathrm{N}_{0}^{\mathrm{b}}(\mu, \mathrm{F})=\mathrm{T}(\mathrm{b}) \mu+\int_{0}^{\mathrm{b}} \mathrm{T}(\mathrm{b}-\mathrm{s}) \mathrm{F}(\mathrm{s}) \mathrm{d} s: \mathrm{X} \times \mathrm{L}_{2}(\mathrm{~J}, \mathrm{X}) \rightarrow \mathrm{X} .
$$


Definition 3.1 ([21]). The system (3.5) is said to be exactly null controllable on $J$ if $\operatorname{ImL}_{0}^{\mathrm{b}} \supset \operatorname{ImN}_{0}^{\mathrm{b}}$ or there exists a $\gamma>0$ such that $\left\|\left(L_{0}^{b}\right)^{*} \mu\right\|^{2} \geqslant \gamma\left\|\left(N_{0}^{b}\right)^{*} \mu\right\|^{2}$ for all $\mu \in X$.

Lemma 3.2 ([19]). Suppose that the linear system (3.5) is exactly null controllable on J. Then the linear operator $\left(\mathrm{L}_{0}\right)^{-1} \mathrm{~N}_{0}^{\mathrm{b}}: \mathrm{X} \times \mathrm{L}_{2}(\mathrm{~J}, \mathrm{X}) \rightarrow \mathrm{L}_{2}(\mathrm{~J}, \mathrm{U})$ is bounded and the control

$$
u(t)=-\left(L_{0}\right)^{-1}\left[T(b) \mu_{0}+\int_{0}^{b} T(b-s) F(s) d s\right](t)
$$

transferees the system (3.5) from $\mu_{0}$ to 0 , where $\mathrm{L}_{0}$ is the restriction of $\mathrm{L}_{0}^{\mathrm{b}}$ to $\left[\mathrm{ker}^{\mathrm{b}}\right]^{\perp}$ and $\mathrm{F} \in \mathrm{L}_{2}(\mathrm{~J}, \mathrm{X})$.

Definition 3.3. We say $x \in \bar{C}$ is a mild solution to (3.1) if it satisfies that

$$
\begin{aligned}
x(t)= & T(t) x_{0}+\int_{0}^{t}[T(t-s) \sigma-A T(t-s)] B u(s) d s+\int_{0}^{t} T(t-s) f_{1}(s, x(s)) d s \\
& +\int_{0}^{t} \int_{0}^{s} T(t-s) f_{2}(\eta, x(\eta)) d \omega(\eta) d s+\int_{0}^{t} T(t-s) g(s, x(s)) d Z_{H}(s), t \in J .
\end{aligned}
$$

Definition 3.4. The system (3.1) is said to be exact null controllable on the interval $\mathrm{J}$ if there exists a stochastic control $u \in \mathrm{L}_{2}(\mathrm{~J}, \mathrm{U})$ such that the solution $x(t)$ of the system (3.1) satisfies $x(b)=0$.

To prove the main result in this section, we need addition hypothesis:

(H8) The linear system (3.5) is exactly null controllable on J.

Theorem 3.5. If the hypotheses ( $\mathrm{H} 1)-(\mathrm{H} 8)$ are satisfied, then the boundary control system (3.1) is exactly null controllable on J provided that

$$
25 \mathrm{M}^{2}\left[\delta+\operatorname{Tr}(\mathrm{Q}) \delta_{1}+2 \mathrm{Hb}^{2 \mathrm{H}-1} \delta_{2}\right]\left[1+\left[\mathrm{M}^{2}\|\sigma\|^{2}+\mathrm{M}_{1}^{2}\right]\|\mathrm{B}\|^{2}\left\|\left(\mathrm{~L}_{0}\right)^{-1}\right\|^{2}\right]<1 .
$$

Proof. For an arbitrary $x(\cdot)$ define the operator $\Phi$ on $\bar{C}$ as follows

$$
\begin{aligned}
(\Phi x)(t)= & T(t) x_{0}+\int_{0}^{t}[T(t-s) \sigma-A T(t-s)] B u(s) d s+\int_{0}^{t} T(t-s) f_{1}(s, x(s)) d s \\
& +\int_{0}^{t} \int_{0}^{s} T(t-s) f_{2}(\eta, x(\eta)) d \omega(\eta) d s+\int_{0}^{t} T(t-s) g(s, x(s)) d Z_{H}(s), t \in J,
\end{aligned}
$$

where

$$
\begin{aligned}
u(t)= & -\left(L_{0}\right)^{-1}\left\{T(b) x_{0}+\int_{0}^{b} T(b-s) f_{1}(s, x(s)) d s+\int_{0}^{b} \int_{0}^{s} T(b-s) f_{2}(\eta, x(\eta)) d \omega(\eta) d s\right. \\
& \left.+\int_{0}^{b} T(b-s) g(s, x(s)) d Z_{H}(s)\right\} .
\end{aligned}
$$

It will be shown that the operator $\Phi$ from $\bar{C}$ into itself has a fixed point. We claim that there exists a positive number $r$ such that $\Phi\left(B_{r}\right) \subseteq B_{r}$. If it is not true, then for each positive number $r$, there is a function $x_{r}(\cdot) \in B_{r}$, but $\Phi\left(x_{r}\right) \notin B_{r}$, that is $\left\|\Phi x_{r}\right\|_{\bar{C}}^{2}>r$ for some $t=t(r) \in J$, where $t(r)$ denotes that $t$ is dependent of $r$.

From our hypotheses, we obtain

$$
\begin{aligned}
r \leqslant\left\|\Phi x_{r}\right\|_{\bar{C}}^{2}= & \sup _{t \in J} E\left\|\Phi\left(x_{r}\right)(t)\right\|^{2} \\
\leqslant & 25 M^{2} E\left\|x_{0}\right\|^{2}+25 M^{2} b\left[M^{2}\|\sigma\|^{2}+M_{1}^{2}\right]\|B\|^{2}\left\|\left(L_{0}\right)^{-1}\right\|^{2} \\
& \times\left[\left\|x_{0}\right\|_{\bar{C}}^{2}+\int_{0}^{b} \rho_{r}(s) d s+\operatorname{Tr}(Q) \int_{0}^{b} h_{r}(s) d s+2 H b^{2 H-1} \int_{0}^{b} k_{r}(s) d s\right] \\
& +25 M^{2} \int_{0}^{t} \rho_{r}(s) d s+25 \operatorname{Tr}(Q) M^{2} \int_{0}^{t} h_{r}(s) d s+50 H b^{2 H-1} M^{2} \int_{0}^{t} k_{r}(s) d s .
\end{aligned}
$$


Dividing both sides of (3.7) by $r$ and taking the lower limit $r \rightarrow+\infty$, we get

$$
25 \mathrm{M}^{2}\left[\delta+\operatorname{Tr}(\mathrm{Q}) \delta_{1}+2 \mathrm{Hb}^{2 \mathrm{H}-1} \delta_{2}\right]\left[1+\left[\mathrm{M}^{2}\|\sigma\|^{2}+\mathrm{M}_{1}^{2}\right]\|\mathrm{B}\|^{2}\left\|\left(\mathrm{~L}_{0}\right)^{-1}\right\|^{2}\right] \geqslant 1 .
$$

This contradicts (3.6). Hence, for positive $r, \Phi\left(B_{r}\right) \subseteq B_{r}$ for positive number $r$. In fact, the operator $\Phi$ maps $B_{r}$ into a compact subset of $B_{r}$. To prove this, we first show that the set $V_{r}(t)=\left\{(\Phi x)(t): x \in B_{r}\right\}$ is a precompact in $X$, for every fixed $t \in J$. This is trivial for $t=0$, since $V_{r}(0)=\left\{x_{0}\right\}$. Let $t, 0<t \leqslant b$, be fixed. For $0<\epsilon<t$, take

$$
\begin{aligned}
\left(\Phi^{\epsilon} x\right)(t)= & T(t) x_{0}+\int_{0}^{t-\epsilon}[T(t-s) \sigma-A T(t-s)] B u(s) d s+\int_{0}^{t-\epsilon} T(t-s) f_{1}(s, x(s)) d s \\
& +\int_{0}^{t-\epsilon} \int_{0}^{s} T(t-s) f_{2}(\eta, x(\eta)) d \omega(\eta) d s+\int_{0}^{t-\epsilon} T(t-s) g(s, x(s)) d Z_{H}(s) \\
= & T(t) x_{0}+T(\epsilon) \int_{0}^{t-\epsilon}[T(t-\epsilon-s) \sigma-A T(t-\epsilon-s)] B u(s) d s+T(\epsilon) \int_{0}^{t-\epsilon} T(t-\epsilon-s) f_{1}(s, x(s)) d s \\
& +T(\epsilon) \int_{0}^{t-\epsilon} \int_{0}^{s} T(t-s) f_{2}(\eta, x(\eta)) d \omega(\eta) d s+T(\epsilon) \int_{0}^{t-\epsilon} T(t-\epsilon-s) g(s, x(s)) d Z_{H}(s) .
\end{aligned}
$$

Since $u(s)$ is bounded and $T(\epsilon)(\epsilon>0)$ is a compact operator then the set $V_{\epsilon}(t)=\left\{\left(\Phi^{\epsilon} x\right)(t): x \in B_{r}\right\}$ is precompact in $X$ for every $\epsilon, 0<\epsilon<t$. Moreover, for every $x \in B_{r}$, we have

$$
\begin{aligned}
& \left\|\Phi x-\Phi^{\epsilon} x\right\|_{\overline{\mathrm{C}}}^{2}=\sup _{\mathrm{t} \in \mathrm{J}} \mathrm{E}\left\|(\Phi x)(\mathrm{t})-\left(\Phi^{\epsilon} \chi\right)(\mathrm{t})\right\|^{2} \\
& \leqslant 25 M^{2} \int_{t-\epsilon}^{t}\left[M^{2}\|\sigma\|^{2}+M_{1}^{2}\right]\|B\|^{2}\left\|\left(L_{0}\right)^{-1}\right\|^{2} \\
& \times\left[E\left\|x_{0}\right\|^{2}+\int_{0}^{b} \rho_{r}(s) d s+\operatorname{Tr}(Q) \int_{0}^{b} h_{r}(s) d s+2 \mathrm{Hb}^{2 \mathrm{H}-1} \int_{0}^{\mathrm{b}} \mathrm{k}_{\mathrm{r}}(\mathrm{s}) \mathrm{ds}\right](\mathrm{s}) \mathrm{d} s \\
& +25 M^{2} \int_{t-\epsilon}^{t} \rho_{r}(s) d s+25 \operatorname{Tr}(Q) M^{2} \int_{t-\epsilon}^{t} h_{r}(s) d s+50 \mathrm{Hb}^{2 H-1} M^{2} \int_{t-\epsilon}^{t} k_{r}(s) d s .
\end{aligned}
$$

We see that for each $x \in \mathrm{B}_{\mathrm{r}},\left\|\Phi x-\Phi^{\epsilon} x\right\|_{\overline{\mathrm{C}}}^{2} \rightarrow 0$ as $\epsilon \rightarrow 0^{+}$. Therefore, there are precompact sets arbitrary close to the set $V_{r}(t)$ and so $V_{r}(t)$ is precompact in $X$.

Next we prove that the family $\left\{\Phi x: x \in B_{r}\right\}$ is an equicontinuous family of functions. Let $x \in B_{r}$ and $t_{1}, t_{2} \in J$ such that $0<t_{1}<t_{2}$, then

$$
\begin{aligned}
&\left\|(\Phi x)\left(t_{2}\right)-(\Phi x)\left(t_{1}\right)\right\|_{\overline{\bar{C}}}^{2} \\
& \leqslant 25\left\|T\left(t_{2}\right)-T\left(t_{1}\right)\right\|^{2}\left\|x_{0}\right\|_{\overline{\mathrm{C}}}^{2}+25 \| \int_{0}^{t_{1}}\left[T\left(t_{2}-s\right) \sigma-A T\left(t_{2}-s\right)-T\left(t_{1}-s\right) \sigma+A T\left(t_{1}-s\right)\right] \\
& \times-\left(L_{0}\right)^{-1}\left\{T(b) x_{0}+\int_{0}^{b} T(b-s) f_{1}(s, x(s)) d s+\int_{0}^{b} \int_{0}^{\eta} T(b-s) f_{2}(\eta, x(\eta)) d \omega(\eta) d s\right. \\
&\left.+\int_{0}^{b} T(b-s) g(s, x(s)) d Z_{H}(s)\right\}(s) d s\left\|_{\overline{\mathrm{C}}}^{2}+25\right\| \int_{t_{1}}^{t_{2}}\left[T\left(t_{2}-s\right) \sigma-A T\left(t_{2}-s\right)\right] \\
& \times-\left(L_{0}\right)^{-1}\left\{T(b) x_{0}+\int_{0}^{b} T(b-s) f_{1}(s, x(s)) d s+\int_{0}^{b} \int_{0}^{\eta} T(b-s) f_{2}(\eta, x(\eta)) d \omega(\eta) d s\right. \\
&\left.+\int_{0}^{b} T(b-s) g(s, x(s)) d Z_{H}(s)\right\}(s) d s \|_{\overline{\mathrm{C}}}^{2} \\
&+25\left\|\int_{0}^{t_{1}}\left[T\left(t_{2}-s\right)-T\left(t_{1}-s\right)\right] \int_{0}^{s} f_{2}(\eta, x(\eta)) d \omega(\eta) d s\right\|_{\overline{\mathrm{C}}}^{2}
\end{aligned}
$$




$$
\begin{aligned}
& +25\left\|\int_{t_{1}}^{t_{2}} T\left(t_{2}-s\right) \int_{0}^{s} f_{2}(\eta, x(\eta)) d \omega(\eta) d s\right\|_{\overline{\bar{C}}}^{2} \\
& +25\left\|\int_{0}^{t_{1}}\left[T\left(t_{2}-s\right)-T\left(t_{1}-s\right)\right] g(s, x(s)) d Z_{H}(s)\right\|_{\bar{C}}^{2} \\
& +25\left\|\int_{t_{1}}^{t_{2}} T\left(t_{2}-s\right) g(s, x(s)) d Z_{H}(s)\right\|_{\overline{\bar{C}}}^{2} .
\end{aligned}
$$

From the above fact, we see that $\left\|(\Phi x)\left(t_{2}\right)-(\Phi x)\left(t_{1}\right)\right\|_{\overline{\mathrm{C}}}^{2}$ tends to zero independently of $x \in B_{r}$ as $t_{2} \rightarrow t_{1}$. Since the compactness of $T(t)$ for $t>0$ implies the continuity in the uniform operator topology. Thus, $\Phi\left(B_{r}\right)$ is both equicontinuous and bounded. By the Arzela-Ascoli theorem $\Phi\left(B_{r}\right)$ is precompact in $X$. Hence $\Phi$ is a completely continuous operator on $X$. From the Schauder fixed point theorem, $\Phi$ has a fixed point in $B_{r}$. Any fixed point of $\Phi$ is a mild solution of (3.1) on J. Therefore the system (3.1) is exact null controllable on J.

\section{Sobolev-type neutral integrodifferential system}

In this section, we investigate the exact null controllability for Sobolev type neutral stochastic integrodifferential system with Rosenblatt process in the following form

$$
\begin{cases}\frac{d}{d t}\left[G x(t)+f_{1}(t, x(t))\right]=\sigma x(t)+\int_{0}^{t} f_{2}(s, x(s)) d \omega(s)+g(t, x(t)) \frac{d Z_{H}(t)}{d t}, & t \in J=[0, b] \\ \tau x(t)=B_{1} u(t), & t \in J=[0, b] \\ x(0)=x_{0}, & \end{cases}
$$

where $G: D(G) \subset X \rightarrow X$ is a linear operator. Let $y(t)=G x(t)$ for $x \in X$, then the equation (4.1) can be written as

$$
\begin{cases}\frac{d}{d t}\left[y(t)+f_{1}\left(t, G^{-1} y(t)\right)\right]=\sigma G^{-1} y(t)+\int_{0}^{t} f_{2}\left(s, G^{-1} y(s)\right) d w(s) & \\ \quad+g\left(t, G^{-1} y(t)\right) \frac{d Z_{H}(t)}{d t}, & t \in J=[0, b], \\ \tau G^{-1} y(t)=B_{1} u(t), & t \in J=[0, b], \\ y(0)=y_{0}, & \end{cases}
$$

where $\tau G^{-1}: X \rightarrow X$ is a linear operator. Let $A G^{-1}: X \rightarrow X$ be a linear operator defined by

$$
\mathrm{D}\left(A \mathrm{G}^{-1}\right)=\left\{\theta \in \mathrm{D}\left(\sigma \mathrm{G}^{-1}\right): \tau \mathrm{G}^{-1} \theta=0\right\}, \quad A G^{-1} \theta=\sigma \mathrm{G}^{-1} \theta \text {, for } \theta \in \mathrm{D}\left(A \mathrm{G}^{-1}\right) \text {. }
$$

The operators $A: D(A) \subset X \rightarrow X$ and $G: D(G) \subset X \rightarrow X$ satisfy the following hypotheses:

(H9) $A$ and $G$ are closed linear operators.

(H10) $D(G) \subset D(A)$ and $G$ is bijective.

$(\mathrm{H} 11) \mathrm{G}^{-1}: \mathrm{X} \rightarrow \mathrm{D}(\mathrm{G})$ is continuous.

Here, (H9) and (H10) together with the closed graph theorem imply the boundedness of the linear operator $A G^{-1}: X \rightarrow X$.

(H12) For each $t \in J$ and for $\lambda \in\left(\rho\left(A G^{-1}\right)\right)$, the resolvent of $A G^{-1}$, the resolvent $R\left(\lambda, A G^{-1}\right)$ is compact operator.

Lemma 4.1 ([28]). Let $T(t)$ be a uniformly continuous semigroup. If the resolvent set $R(\lambda, A)$ of $A$ is compact for every $\lambda \in \rho(A)$, then $T(t)$ is a compact semigroup. 
From the above fact, $A G^{-1}$ generates a compact semigroup $\{S(t), t>0\}$ in $X$, which means that there exists $M>1$ such that $\sup _{t \in J}\|S(t)\| \leqslant M$. We suppose that $0 \in \rho\left(A G^{-1}\right)$, the resolvent set of $A Z^{-1}$, for every $t>0$. We define the fractional power $\left(A G^{-1}\right)^{-\gamma}$ by

$$
\left(A G^{-1}\right)^{-\gamma}=\frac{1}{\Gamma(\gamma)} \int_{0}^{\infty} t^{\gamma-1} S(t) d t, \quad \gamma>0
$$

For $\gamma \in(0,1],\left(A G^{-1}\right)^{\gamma}$ is a closed linear operator on its domain $\mathrm{D}\left(\left(A G^{-1}\right)^{\gamma}\right)$. Furthermore, the subspace $\mathrm{D}\left(\left(A G^{-1}\right)^{\gamma}\right)$ is dense in $X$. We will introduce the following basic properties of $\left(A G^{-1}\right)^{\gamma}$.

Theorem 4.2 ([31]).

(1) Let $0<\gamma \leqslant 1$, then $X_{\gamma}:=\mathrm{D}\left(\left(A G^{-1}\right)^{\gamma}\right)$ is a Banach space with the norm $\|x\|_{\gamma}=\left\|\left(A G^{-1}\right)^{\gamma} x\right\|, x \in X_{\gamma}$.

(2) If $0<\beta<\gamma \leqslant 1$, then $\mathrm{D}\left(\left(A G^{-1}\right)^{\gamma}\right) \hookrightarrow \mathrm{D}\left(\left(A G^{-1}\right)^{\beta}\right)$ and the embedding is compact whenever the resolvent operator of $\left(\mathrm{AG}^{-1}\right)$ is compact.

(3) For every $0<\gamma \leqslant 1$, there exists a positive constant $\mathrm{C}_{\gamma}$ such that

$$
\left\|\left(A G^{-1}\right)^{\gamma} S(t)\right\| \leqslant \frac{C_{\gamma}}{t^{\gamma}}, 0<t \leqslant b .
$$

Let $y(t)$ be the solution of the system (4.2). Then we can define a function $z(t)=y(t)-B u(t)$ and from our assumption we see that $z(t) \in D\left(A G^{-1}\right)$. Hence (4.2) can be written in terms of $A$ and $B$ as

$$
\left\{\begin{array}{l}
\frac{d}{d t}\left[y(t)+f_{1}\left(t, G^{-1} y(t)\right)\right]=A G^{-1} z(t)+\sigma G^{-1} B u(t)+\int_{0}^{t} f_{2}\left(s, G^{-1} y(s)\right) d \omega(s) \\
\quad+g\left(t, G^{-1} y(t)\right) \frac{d Z_{H}(t)}{d t}, t \in J \\
y(t)=z(t)+B u(t) \\
y(0)=y_{0}
\end{array}\right.
$$

If $u$ is continuously differentiable on $[0, b]$, then $z$ can be defined as a mild solution to the Cauchy problem

$$
\left\{\begin{array}{l}
\frac{d}{d t}\left[z(t)+f_{1}\left(t, G^{-1} y(t)\right)\right]=A G^{-1} z(t)+\sigma G^{-1} B u(t)-B \frac{d u}{d t}+\int_{0}^{t} f_{2}\left(s, G^{-1} y(s)\right) d w(s) \\
\quad+g\left(t, G^{-1} y(t)\right) \frac{d Z_{H}(t)}{d t}, t \in J \\
z(0)=y(0)-B u(0) .
\end{array}\right.
$$

Then the mild solution of (4.2) is given by

$$
\begin{aligned}
y(t)= & S(t)\left[y(0)+f_{1}\left(0, G^{-1} y(0)\right)-B u(0)\right]-f_{1}\left(t, G^{-1} y(t)\right)+\int_{0}^{t} A G^{-1} S(t-s) f_{1}\left(s, G^{-1} y(s)\right) \\
& +B u(t)+\int_{0}^{t} S(t-s)\left[\sigma G^{-1} B u(s)-B \frac{d u(s)}{d s}\right] d s \\
& +\int_{0}^{t} \int_{0}^{s} S(t-s) f_{2}\left(\eta, G^{-1} y(\eta)\right) d w(\eta) d s+\int_{0}^{t} S(t-s) g\left(s, G^{-1} y(s)\right) d Z_{H}(s) .
\end{aligned}
$$

By using integration by parts, we obtain

$$
\begin{aligned}
y(t)= & S(t)\left[y(0)+f_{1}\left(0, G^{-1} y(0)\right)\right]-f_{1}\left(t, G^{-1} y(t)\right)+\int_{0}^{t} A G^{-1} S(t-s) f_{1}\left(s, G^{-1} y(s)\right) \\
& +\int_{0}^{t}\left[S(t-s) \sigma G^{-1} B-A G^{-1} S(t-s) B\right] u(s) d s \\
& +\int_{0}^{t} \int_{0}^{s} S(t-s) f_{2}\left(\eta, G^{-1} y(\eta)\right) d \omega(\eta) d s+\int_{0}^{t} S(t-s) g\left(s, G^{-1} y(s)\right) d Z_{H}(s) .
\end{aligned}
$$


Hence the mild solution of system (4.1) is given by

$$
\begin{aligned}
x(t)= & G^{-1} S(t)\left[G x(0)+f_{1}(0, x(0))\right]-f_{1}(t, x(t))+\int_{0}^{t} G^{-1} A G^{-1} S(t-s) f_{1}(s, x(s)) \\
& +\int_{0}^{t} G^{-1}\left[S(t-s) \sigma G^{-1} B-A G^{-1} S(t-s) B\right] u(s) d s \\
& +\int_{0}^{t} \int_{0}^{s} S(t-s) G^{-1} f_{2}(\eta, x(\eta)) d \omega(\eta) d s+\int_{0}^{t} G^{-1} S(t-s) g(s, x(s)) d Z_{H}(s) .
\end{aligned}
$$

To study the exact null controllability of (4.1), we consider the Soblev-type linear boundary control system

$$
\begin{cases}\frac{d}{d t}[G \mu(t)]=\sigma \mu(t)+F(t), & t \in J=[0, b], \\ \tau \mu(t)=B_{1} u(t), & t \in J=[0, b], \\ \mu(0)=\mu_{0}, & \end{cases}
$$

associated with the system (4.1). Consider

$$
\mathrm{L}_{0}^{\mathrm{b}} \mathrm{u}=\int_{0}^{\mathrm{b}} \mathrm{G}^{-1}\left[\mathrm{~T}(\mathrm{~b}-\mathrm{s}) \sigma \mathrm{G}^{-1} \mathrm{~B}-\mathrm{AG} \mathrm{G}^{-1} \mathrm{~T}(\mathrm{~b}-\mathrm{s}) \mathrm{B}\right] \mathrm{u}(\mathrm{s}) \mathrm{d} s: \mathrm{L}_{2}(\mathrm{~J}, \mathrm{U}) \rightarrow \mathrm{X},
$$

where $L_{0}^{b} u$ has a bounded inverse operator $\left(L_{0}\right)^{-1}$ with values in $L_{2}(J, U) / \operatorname{ker}\left(L_{0}^{b}\right)$, and

$$
N_{0}^{b}(\mu, F)=G^{-1} T(b) G \mu+\int_{0}^{b} G^{-1} T(b-s) F(s) d s: X \times L_{2}(J, X) \rightarrow X
$$

Definition 4.3. The system (4.3) is said to be exactly null controllable on $\mathrm{J}$ if $\operatorname{ImL}_{0}^{\mathrm{b}} \supset \operatorname{Im}_{0}^{\mathrm{b}}$ or there exists a $\gamma>0$ such that $\left\|\left(\mathrm{L}_{0}^{\mathrm{b}}\right)^{*} \mu\right\|^{2} \geqslant \gamma\left\|\left(\mathrm{N}_{0}^{\mathrm{b}}\right)^{*} \mu\right\|^{2}$ for all $\mu \in \mathrm{X}$.

Lemma 4.4. Suppose that the linear system (4.3) is exactly null controllable on J. Then the linear operator $\left(\mathrm{L}_{0}\right)^{-1} \mathrm{~N}_{0}^{\mathrm{b}}: \mathrm{X} \times \mathrm{L}_{2}(\mathrm{~J}, \mathrm{X}) \rightarrow \mathrm{L}_{2}(\mathrm{~J}, \mathrm{U})$ is bounded and the control

$$
u(t)=-\left(L_{0}\right)^{-1}\left[G^{-1} T(b) G \mu_{0}+\int_{0}^{b} G^{-1} T(b-s) F(s) d s\right](t)
$$

transferees the system (4.3) from $\mu_{0}$ to 0 , where $\mathrm{L}_{0}$ is the restriction of $\mathrm{L}_{0}^{\mathrm{b}}$ to $\left[\mathrm{ker}^{\mathrm{b}}\right]^{\perp}$ and $\mathrm{F} \in \mathrm{L}_{2}(\mathrm{~J}, \mathrm{X})$.

Definition 4.5. We say $x \in \bar{C}$ is a mild solution to (4.1) if it satisfies that

$$
\begin{aligned}
x(t)= & G^{-1} S(t)\left[G x(0)+f_{1}(0, x(0))\right]-G^{-1} f_{1}(t, x(t))+\int_{0}^{t} G^{-1} A G^{-1} S(t-s) f_{1}(s, x(s)) \\
& +\int_{0}^{t} G^{-1}\left[S(t-s) \sigma G^{-1} B-A G^{-1} S(t-s) B\right] u(s) d s \\
& +\int_{0}^{t} \int_{0}^{s} S(t-s) G^{-1} f_{2}(\eta, x(\eta)) d \omega(\eta) d s+\int_{0}^{t} G^{-1} S(t-s) g(s, x(s)) d Z_{H}(s) .
\end{aligned}
$$

Definition 4.6. The system (4.1) is said to be exact null controllable on the interval $\mathrm{J}$ if there exists a stochastic control $u \in L_{2}(J, U)$ such that the solution $x(t)$ of the system (4.1) satisfies $x(b)=0$.

To prove the main result in this section, we need addition hypotheses.

(H13) For all $t \in(0, b]$ and $u \in U, S(t) B u \in D\left(A G^{-1}\right)$. Moreover, there exists a positive constant $C>0$ such that $\left.\| A G^{-1}\right) S(t) \| \leqslant C$.

(H14) The linear system (4.3) is exactly null controllable on J. 
(H15) $f_{1}: J \times X \rightarrow X$ is a continuous function, and there exists a constant $\frac{1}{2}<\beta<1$ and $M_{1}, M_{2}>0$ such that for all $t \in J$ and $x, y \in X$ the following inequalities are satisfied:

$$
\begin{array}{r}
E\left\|\left(A G^{-1}\right)^{\beta} f_{1}(t, x)-\left(A G^{-1}\right)^{\beta} f_{1}(t, y)\right\|^{2} \leqslant M_{1} E\|x-y\|^{2}, \\
E\left\|\left(A G^{-1}\right)^{\beta} f_{1}(t, x)\right\|^{2} \leqslant M_{2}\left(1+E\|x\|^{2}\right) .
\end{array}
$$

Theorem 4.7. If the hypotheses (H1), (H2), (H6), (H7), and (H9)-(H15) are satisfied, then the boundary control system (4.1) is exactly null controllable on J provided that

$$
\begin{aligned}
& {\left[M_{2}\left[M_{0}^{2}+\frac{C_{1-\beta}^{2} b^{2 \beta-1}\left\|G^{-1}\right\|^{2}}{2 \beta-1}\right]+\operatorname{Tr}(Q) M^{2}\left\|G^{-1}\right\|^{2} \delta_{1}+2 H^{2}\left\|G^{-1}\right\|^{2} b^{2 H-1} \delta_{2}\right]} \\
& \times 36\left[1+\left\|G^{-1}\right\|^{2} b\left[M^{2}\|\sigma\|^{2}\left\|G^{-1}\right\|^{2}+C^{2}\right]\|B\|^{2}\left\|\left(L_{0}\right)^{-1}\right\|^{2}\right] \leqslant 1, \\
& M_{0}=\left\|\left(A G^{-1}\right)^{-\beta}\right\| \text {. }
\end{aligned}
$$

Proof. For an arbitrary $x(\cdot)$ define the operator $\Psi$ on $\bar{C}$ as follows

$$
\begin{aligned}
(\Psi x)(t)= & G^{-1} S(t)\left[G x(0)+f_{1}(0, x(0))\right]-f_{1}(t, x(t))+\int_{0}^{t} G^{-1} A G^{-1} S(t-s) f_{1}(s, x(s)) d s \\
& +\int_{0}^{t} G^{-1}\left[S(t-s) \sigma G^{-1} B-A G^{-1} S(t-s) B\right] u(s) d s \\
& +\int_{0}^{t} \int_{0}^{s} S(t-s) G^{-1} f_{2}(\eta, x(\eta)) d \omega(\eta) d s+\int_{0}^{t} G^{-1} S(t-s) g(s, x(s)) d Z_{H}(s), t \in J,
\end{aligned}
$$

where

$$
\begin{aligned}
u(t)= & -\left(L_{0}\right)^{-1}\left\{G^{-1} S(b)\left[G \times(0)+f_{1}(0, x(0))\right]-f_{1}(b, x(b))+\int_{0}^{b} G^{-1} A G^{-1} S(b-s) f_{1}(s, x(s)) d s\right. \\
& \left.+\int_{0}^{b} \int_{0}^{s} S(b-s) G^{-1} f_{2}(\eta, x(\eta)) d \omega(\eta) d s+\int_{0}^{b} G^{-1} S(b-s) g(s, x(s)) d Z_{H}(s)\right\} .
\end{aligned}
$$

It will be shown that the operator $\Psi$ from $\bar{C}$ into itself has a fixed point. We claim that there exists a positive number $r$ such that $\Psi\left(B_{r}\right) \subseteq B_{r}$. If it is not true, then for each positive number $r$, there is a function $x_{r}(\cdot) \in B_{r}$, but $\Psi\left(x_{r}\right) \notin B_{r}$, that is $\left\|\Psi x_{r}\right\|_{\bar{C}}^{2}>r$ for some $t=t(r) \in J$, where $t(r)$ denotes that $t$ is dependent of $r$. From our hypotheses, we obtain

$$
\begin{aligned}
r \leqslant & \left\|\Psi x_{r}\right\|_{\bar{C}}^{2} \\
= & \sup _{t \in J} E\left\|\Psi\left(x_{r}\right)(t)\right\|^{2} \\
\leqslant & 36\left\|G^{-1}\right\|^{2}\left[M^{2}\|G\|^{2} E\left\|x_{0}\right\|^{2}+M_{2} C_{-\beta}^{2} b^{2 \beta}\left(1+E\left\|x_{0}\right\|^{2}\right)\right]+\left[M_{0}^{2}+\frac{C_{1-\beta}^{2} b^{2 \beta-1}\left\|G^{-1}\right\|^{2}}{2 \beta-1}\right] 36 M_{2}(1+r) \\
& +36\left\|G^{-1}\right\|^{2} b\left[M^{2}\|\sigma\|^{2}\left\|G^{-1}\right\|^{2}+C^{2}\right]\|B\|^{2}\left\|\left(L_{0}\right)^{-1}\right\|^{2}\left[\left\|G^{-1}\right\|^{2}\left[M^{2}\|G\|^{2} E\left\|x_{0}\right\|^{2}+M_{2} C_{-\beta}^{2} b^{2 \beta}\left(1+E\left\|x_{0}\right\|^{2}\right)\right]\right. \\
& \left.+\left[M_{0}^{2}+\frac{C_{1-\beta}^{2} b^{2 \beta-1}\left\|G^{-1}\right\|^{2}}{2 \beta-1}\right] M_{2}(1+r)+\operatorname{Tr}(Q) M^{2}\left\|G^{-1}\right\|^{2} \int_{0}^{b} h_{r}(s) d s+2 H M^{2}\left\|G^{-1}\right\|^{2} b^{2 H-1} \int_{0}^{b} k_{r}(s) d s\right] \\
& +36 M^{2}\left\|G^{-1}\right\|^{2} \operatorname{Tr}(Q) \int_{0}^{t} h_{r}(s) d s+72 H M^{2}\left\|G^{-1}\right\|^{2} b^{2 H-1} \int_{0}^{t} k_{r}(s) d s .
\end{aligned}
$$

Dividing both sides of above equation by $r$ and taking the lower limit $r \rightarrow+\infty$, we get

$$
\left[M_{2}\left[M_{0}^{2}+\frac{C_{1-\beta}^{2} b^{2 \beta-1}\left\|G^{-1}\right\|^{2}}{2 \beta-1}\right]+\operatorname{Tr}(Q) M^{2}\left\|G^{-1}\right\|^{2} \delta_{1}+2 H^{2}\left\|G^{-1}\right\|^{2} b^{2 H-1} \delta_{2}\right]
$$




$$
\times 36\left[1+\left\|G^{-1}\right\|^{2} b\left[M^{2}\|\sigma\|^{2}\left\|G^{-1}\right\|^{2}+C^{2}\right]\|B\|^{2}\left\|\left(L_{0}\right)^{-1}\right\|^{2}\right] \geqslant 1 .
$$

This contradicts (4.4). Hence, for positive $r, \Psi\left(B_{r}\right) \subseteq B_{r}$ for positive number $r$. In fact, the operator $\Psi$ maps $B_{r}$ into a compact subset of $B_{r}$. To prove this, we first show that the set $V_{r}(t)=\left\{\left(\Psi_{x}\right)(t): x \in B_{r}\right\}$ is a precompact in $X$, for every fixed $t \in J$. This is trivial for $t=0$, since $V_{r}(0)=\left\{x_{0}\right\}$. Let $t, 0<t \leqslant b$, be fixed. For $0<\epsilon<t$, take

$$
\begin{aligned}
\left(\Psi^{\epsilon} x\right)(t)= & G^{-1} S(t)\left[G x(0)+f_{1}(0, x(0))\right]-f_{1}(t, x(t))+\int_{0}^{t-\epsilon} G^{-1} A G^{-1} S(t-s) f_{1}(s, x(s)) d s \\
& +\int_{0}^{t-\epsilon} G^{-1}\left[S(t-s) \sigma G^{-1} B-A G^{-1} S(t-s) B\right] u(s) d s \\
& +\int_{0}^{t-\epsilon} \int_{0}^{s} S(t-s) G^{-1} f_{2}(\eta, x(\eta)) d \omega(\eta) d s+\int_{0}^{t-\epsilon} G^{-1} S(t-s) g(s, x(s)) d Z_{H}(s), t \in J \\
= & G^{-1} S(t)\left[G x(0)+f_{1}(0, x(0))\right]-f_{1}(t, x(t))+S(\epsilon) \int_{0}^{t-\epsilon} G^{-1} A G^{-1} S(t-\epsilon-s) f_{1}(s, x(s)) d s \\
& +S(\epsilon) \int_{0}^{t-\epsilon} G^{-1}\left[S(t-\epsilon-s) \sigma G^{-1} B-A G^{-1} S(t-\epsilon-s) B\right] u(s) d s \\
& +S(\epsilon) \int_{0}^{t-\epsilon} \int_{0}^{s} S(t-\epsilon-s) G^{-1} f_{2}(\eta, x(\eta)) d \omega(\eta) d s \\
& +S(\epsilon) \int_{0}^{t-\epsilon} G^{-1} S(t-\epsilon-s) g(s, x(s)) d Z_{H}(s), t \in J .
\end{aligned}
$$

Since $u(s)$ is bounded and $S(\epsilon)(\epsilon>0)$ is a compact operator, then the set $V_{\epsilon}(t)=\left\{\left(\Psi^{\epsilon} x\right)(t): x \in B_{r}\right\}$ is precompact in $X$ for every $\epsilon, 0<\epsilon<t$. Moreover, for every $x \in B_{r}$, we have

$$
\begin{aligned}
\left\|\Psi x-\Psi^{\epsilon} x\right\|_{\overline{\mathrm{C}}}^{2}= & \sup _{\mathrm{t} \in \mathrm{J}} \mathrm{E}\left\|(\Psi x)(\mathrm{t})-\left(\Psi^{\epsilon} x\right)(\mathrm{t})\right\|^{2} \\
\leqslant & 36 \int_{t-\epsilon}^{t} G^{-1} A G^{-1} S(t-s) f_{1}(s, x(s)) d s+36 \int_{t-\epsilon}^{t}\left\|G^{-1}\right\|^{2}\left[M^{2}\|\sigma\|^{2}\left\|G^{-1}\right\|^{2}+C^{2}\right]\|B\|^{2}\left\|\left(L_{0}\right)^{-1}\right\|^{2} \\
& \times\left\{\left\|G^{-1}\right\|^{2}\left[M^{2}\|G\|^{2} E\left\|x_{0}\right\|^{2}+M_{2} C_{-\beta}^{2} b^{2 \beta}\left(1+E\left\|x_{0}\right\|^{2}\right)\right]+\operatorname{Tr}(Q) \int_{0}^{b} h_{r}(s) d s\right. \\
& \left.+2 \mathrm{Hb}^{2 H-1} \int_{0}^{b} k_{r}(s) d s\right\}(s) d s+36 \operatorname{Tr}(Q) M^{2} \int_{t-\epsilon}^{t} h_{r}(s) d s+72 H b^{2 H-1} M^{2} \int_{t-\epsilon}^{t} k_{r}(s) d s .
\end{aligned}
$$

We see that for each $x \in B_{r},\left\|\Psi x-\Psi^{\epsilon} x\right\|_{\overline{\mathrm{C}}}^{2} \rightarrow 0$ as $\epsilon \rightarrow 0^{+}$. Therefore, there are precompact sets arbitrary close to the set $V_{r}(t)$ and so $V_{r}(t)$ is precompact in $X$.

Next we prove that the family $\left\{\Psi_{x}: x \in B_{r}\right\}$ is an equicontinuous family of functions. Let $x \in B_{r}$ and $t_{1}, t_{2} \in J$ such that $0<t_{1}<t_{2}$, then

$$
\begin{aligned}
\|(\Psi x)\left(t_{2}\right) & -(\Psi x)\left(t_{1}\right) \|_{\overline{\mathrm{C}}}^{2} \\
\leqslant & 36\left\|G^{-1}\right\|^{2}\left\|S\left(t_{2}\right)-S\left(t_{1}\right)\right\|^{2}\left\|G x(0)+f_{1}(0, x(0))\right\|_{\bar{C}}^{2}+36\left\|f\left(t_{2}, x\left(t_{2}\right)\right)-f\left(t_{1}, x\left(t_{1}\right)\right)\right\|_{\bar{C}}^{2} \\
& +36 \| \int_{0}^{t_{1}} G^{-1}\left[S\left(t_{2}-s\right) \sigma G^{-1}-A G^{-1} S\left(t_{2}-s\right)-S\left(t_{1}-s\right) \sigma G^{-1}+A G^{-1} S\left(t_{1}-s\right)\right] \\
& \times-\left(L_{0}\right)^{-1}\left\{T(b) x_{0}+\int_{0}^{b} T(b-s) f_{1}(s, x(s)) d s+\int_{0}^{b} \int_{0}^{\eta} T(b-s) f_{2}(\eta, x(\eta)) d \omega(\eta) d s\right. \\
& \left.+\int_{0}^{b} T(b-s) g(s, x(s)) d Z_{H}(s)\right\}(s) d s\left\|_{\bar{C}}^{2}+25\right\| \int_{t_{1}}^{t_{2}}\left[T\left(t_{2}-s\right) \sigma-A T\left(t_{2}-s\right)\right] \\
& \times-\left(L_{0}\right)^{-1}\left\{G^{-1} S(b)\left[G x(0)+f_{1}(0, x(0))\right]-f_{1}(b, x(b))+\int_{0}^{b} G^{-1} A G^{-1} S(b-s) f_{1}(s, x(s)) d s\right.
\end{aligned}
$$




$$
\begin{aligned}
& \left.+\int_{0}^{b} \int_{0}^{\eta} S(b-s) G^{-1} f_{2}(\eta, x(\eta)) d \omega(\eta) d s+\int_{0}^{b} S(b-s) G^{-1} g(s, x(s)) d Z_{H}(s)\right\}(s) d s \|_{\bar{C}}^{2} \\
& +36\left\|\int_{0}^{t_{1}}\left[S\left(t_{2}-s\right)-S\left(t_{1}-s\right)\right] G^{-1} \int_{0}^{s} f_{2}(\eta, x(\eta)) d \omega(\eta) d s\right\|_{\bar{C}}^{2} \\
& +36\left\|\int_{t_{1}}^{t_{2}} S\left(t_{2}-s\right) G^{-1} \int_{0}^{s} f_{2}(\eta, x(\eta)) d \omega(\eta) d s\right\|_{\bar{C}}^{2} \\
& +36\left\|\int_{0}^{t_{1}}\left[S\left(t_{2}-s\right)-S\left(t_{1}-s\right)\right] G^{-1} g(s, x(s)) d Z_{H}(s)\right\|_{\bar{C}}^{2}+36\left\|\int_{t_{1}}^{t_{2}} S\left(t_{2}-s\right) G^{-1} g(s, x(s)) d Z_{H}(s)\right\|_{\bar{C}}^{2} .
\end{aligned}
$$

From the above fact, we see that $\left\|(\Psi \chi)\left(t_{2}\right)-(\Psi \chi)\left(t_{1}\right)\right\|_{\overline{\mathrm{C}}}^{2}$ tends to zero independently of $x \in B_{r}$ as $t_{2} \rightarrow t_{1}$. Since the compactness of $S(t)$ for $t>0$ implies the continuity in the uniform operator topology. Thus, $\Psi\left(B_{r}\right)$ is both equicontinuous and bounded. By the Arzela-Ascoli theorem $\Psi\left(B_{r}\right)$ is precompact in $X$. Hence $\Psi$ is a completely continuous operator on $X$. From the Schauder fixed point theorem, $\Psi$ has a fixed point in $B_{r}$. Any fixed point of $\Phi$ is a mild solution of (4.1) on J. Therefore the system (4.1) is exact null controllable on J.

\section{Application}

Consider the following boundary control stochastic integro-partial differential system with Rosenblatt process of the form,

$$
\begin{cases}\frac{\partial x(t, y)}{\partial t}=\Delta x(t, y)+\cos (x(t, y))+\int_{0}^{t} \tan ^{-1}(x(s, y)) d \omega(s)+e^{-x(t, y)} \frac{d Z_{H}(t)}{d t}, & \text { in } \bar{Q}=(0, b) \times \Pi, \\ x(t, 0)=u(t, 0), & \text { on } \Sigma=(0, b) \times \Gamma, t \in J, \\ x(t, y)=0, x(0, y)=x_{0}(y), & \text { for } y \in \Pi,\end{cases}
$$

where $\Pi$ is a bounded and open subset of $R^{n}$ with sufficiently smooth boundary $\Gamma$. Let $x_{0} \in L_{2}(\Pi), \omega(t)$ is Wiener process, $Z_{H}$ is a Rosenblatt process with parameter $H \in\left(\frac{1}{2}, 1\right)$ and $u \in L_{2}(\Sigma)$. The functions are $x(t)(y)=x(t, y), f_{1}(t, x(t))(y)=\cos (x(t, y)), f_{2}(s, x(s))(y)=\tan ^{-1}(x(s, y))$, and $g(t, x(t))(y)=e^{-x(t, y)}$. Let $\mathrm{X}=\mathrm{Y}=\mathrm{K}=\mathrm{L}_{2}(\Pi), \mathrm{U}=\mathrm{L}_{2}(\Gamma) \mathrm{B}_{1}=\mathrm{I}$, the identity operator and $\sigma x=\Delta x$ with domain $\mathrm{D}(\sigma)=\{x \in$ $\left.\mathrm{L}_{2}(\Pi): \Delta \mathrm{x} \in \mathrm{L}_{2}(\Pi)\right\}$.

The operator $\theta$ is the trace operator such that $\theta x=\left.x\right|_{\Gamma}$ is well defined and belongs to $H^{-1 / 2}(\Gamma)$ for each $x \in \mathrm{D}(\sigma)$.

Define the operator $A: D(A) \subset X \rightarrow X$ is given by $A x=\Delta x$ with domain $D(A)=H_{0}^{1}(\Omega) \cup H^{2}(\Omega)$, where $H^{k}(\Omega), H^{\beta}(\Gamma)$ are usual Sobolev space on $\Omega, \Gamma$. Then $A$ can be written as

$$
A x=\sum_{n=1}^{\infty} n^{2}\left(x, x_{n}\right) x_{n}, \quad z \in D(A)
$$

where $x_{n}(y)=\sqrt{2} \sin n y, n=1,2,3, \ldots$, is the orthogonal set of eigenvectors of $A$. Furthermore, for $x \in X$

$$
\mathrm{T}(\mathrm{t}) \mathrm{x}=\sum_{n=1}^{\infty} e^{\frac{\mathrm{n}^{2}}{1+\mathrm{n}^{2}} \mathrm{t}}\left(x, x_{\mathrm{n}}\right) x_{\mathrm{n}} .
$$

We define the linear operator $\mathrm{B}: \mathrm{L}_{2}(\Gamma) \rightarrow \mathrm{L}_{2}(\Omega)$ by $\mathrm{Bu}=v_{\mathfrak{u}}$, where $v_{\mathfrak{u}}$ is the unique solution to the Dirichlet boundary value problem,

$$
\begin{aligned}
\Delta v_{\mathrm{u}} & =0, & & \text { in } \Omega, \\
v_{\mathrm{u}} & =\mathrm{u}, & & \text { in } \Gamma .
\end{aligned}
$$

Choose $\mathrm{b}$ and other constants such that all the hypotheses of Theorem 3.5 are satisfied and

$$
25 \mathrm{M}^{2}\left[\delta+\operatorname{Tr}(\mathrm{Q}) \delta_{1}+2 \mathrm{Hb}^{2 \mathrm{H}-1} \delta_{2}\right]\left[1+\left[\mathrm{M}^{2}\|\sigma\|^{2}+\mathrm{M}_{1}^{2}\right]\|\mathrm{B}\|^{2}\left\|\left(\mathrm{~L}_{0}\right)^{-1}\right\|^{2}\right]<1,
$$

so system (5.1) is exactly null controllable on J. 


\section{Conclusion}

Stochastic differential systems with Rosenblatt process and control on the boundary are introduced. By using fractional calculus and stochastic analysis, the sufficient conditions for null boundary controllability of nonlinear integrodifferential system with Rosenblatt process and Sobolev type neutral stochastic integrodifferential system with Rosenblatt process are established. Moreover, we provided an example to illustrate our results.

For future work, we can present the null controllability of noninstantaneous impulsive fractional stochastic evolution inclusions.

\section{Acknowledgements}

We would like to thank the referees and the editor for their important comments and suggestions, which have significantly improved the paper.

\section{References}

[1] T. Abdeljawad, S. Rashid, Z. Hammouch, İ. İscan, Y.-M. Chu, Some new Simpson-type inequalities for generalized p-convex function on fractal sets with applications, Adv. Difference Equ., 2020 (2020), 26 pages. 1

[2] P. Abry, V. Pipiras, Wavelet-based synthesis of the Rosenblatt process, Signal Process, 86 (2006), 2326-2339. 1

[3] H. M. Ahmed, Controllability of fractional stochastic delay equations, Lobachevskii J. Math., 30 (2009), 195-202.

[4] H. M. Ahmed, Boundary controllability of nonlinear fractional integrodifferential systems,Adv. Difference Equ., 2010 (2010), 9 pages. 1

[5] H. M. Ahmed, Approximate controllability of impulsive neutral stochastic differential equations with fractional Brownian motion in a Hilbert space, Adv. Difference Equ., 2014 (2014), 11 pages. 1 1

[6] H. M. Ahmed, Boundary controllability of impulsive nonlinear fractional delay integro-differential system, Cogent Eng., 3 (2016), 15 pages. 1

[7] J. M. P. Albin, A note on the Rosenblatt distributions, Statist. Probab. Lett., 40 (1998), 83-91. 1

[8] G. Arthi, J. H. Park, H. Y. Jung, Existence and exponential stability for neutral stochastic integrodifferential equations with impulses driven by a fractional Brownian motion, Commun. Nonlinear Sci. Numer. Simul., 32 (2016), 145-157. 1

[9] A. Babaei, H. Jafari, S. Banihashemi, Correction to: A numerical scheme to solve a class of two-dimensional nonlinear time-fractional diffusion equations of distributed order, Engineering with Computers, 2021 (2021), 11 pages. 1

[10] K. Balachandran, E. R. Anandhi, Boundary controllability of delay integrodifferential systems in Banach spaces, J. Korean Soc. indust. Appl. Math., 4 (2000), 67-75. 1

[11] K. Balachandran, E. R. Anandhi, Boundary controllability of integrodifferential systems in Banach spaces, Proc. Indian Acad. Sci. Math. Sci., 111 (2001), 127-135. 1

[12] K. Balachandran, P. Balasubramaniam, J. P. Dauer, Local null controllability of nonlinear functional differential systems in Banach space, J. Optim. Theory Appl., 88 (1996), 61-75. 1

[13] K. Balachandran, J.-H. Kim, Sample controllability of nonlinear stochastic integrodifferential systems, Nonlinear Anal. Hybrid Syst., 4 (2010), 543-549. 1

[14] V. Barbu, Boundary control problems with convex cost criterion, SIAM J. Control Optim., 18 (1980), 227-243. 1

[15] A. Boudaoui, T. Caraballo, A. Ouahab, Impulsive neutral functional differential equations driven by a fractional Brownian motion with unbounded delay, Appl. Anal., 95 (2016), 2039-2062. 1

[16] B. Boufoussi, S. Hajji, Neutral stochastic functional differential equations driven by a fractional Brownian motion in a Hilbert space, Statist. Probab. Lett., 82 (2012), 1549-1558. 1

[17] S.-B. Chen, S. Rashid, Z. Hammouch, M. A. Noor, R. Ashraf, Y.-M. Chu, Integral inequalities via Raina's fractional integrals operator with respect to a monotone function, Adv. Difference Equ., 2020 (2020), 20 pages. 1

[18] J. P. Dauer, P. Balasubramaniam, Null controllability of semilinear integrodifferential systems in Banach spaces, Appl. Math. Lett., 10 (1997), 117-123. 1

[19] J. P. Dauer, N. I. Mahmudov, Exact null controllability of semilinear integrodifferential systems in Hilbert spaces, J. Math. Anal. Appl., 299 (2004), 322-332. 1, 3.2

[20] M. A. Diop, K. Ezzinbi, M. M. Mbaye, Existence and global attractiveness of a pseudo almost periodic solution in p-th mean sense for stochastic evolution equation driven by a fractional Brownian motion, Stochastics, 87 (2015), 1061-1093. 1

[21] X. L. Fu, Y. Zhang, Exact null controllability of non-autonomous functional evolution systems with nonlocal conditions, Acta Math. Sci. Ser. B (Engl. Ed.), 33 (2013), 747-757. 1, 3.1

[22] J. Lagnese, Boundary value control of a class of hyperbolic equations in a general region, SIAM J. Control Optim., 15 (1977), 973-983. 1 
[23] E. H. Lakhel, M. McKibben, Controllability for time-dependent neutral stochastic functional differential equations with Rosenblatt process and impulses, Int. J. Control Automa. Syst., 17 (2019), 286-297. 2.1

[24] J.-L. Lions, E. Magenes, Non-homogeneous boundary value problems and applications, Spriner-Verlag, New York, (1972). 1

[25] R. Mabel Lizzy, K. Balachandran, Boundary controllability of nonlinear stochastic fractional system in Hilbert space, Int. J. Appl. Math. Comput. Sci., 28 (2018), 123-133. 1

[26] A. Manouchehrian, A. HaghBin, H. Jafari, Bivariate Generalized Taylor's Formula and Its Applications to Solve FPDEs, Int. J. Appl. Comput. Math., 7 (2021), 11 pages. 1

[27] M. Palanisamy, R. Chinnathambi, Approximate boundary controllability of Sobolev-type stochastic differential systems, J. Egyptian Math. Soc., 22 (2014), 201-208. 1

[28] A. Pazy, Semigroups of Linear Operators and Applications to Partial Differential Equations, Springer-Verlag, New York, (1983). 2, 4.1

[29] V. Pipiras, Wavelet-type expansion of the Rosenblatt process, J. Fourier Anal. Appl., 10 (2004), 599-634. 1

[30] S. Rashid, Z. Hammouch, R. Ashraf, Y.-M. Chu, New computation of unified bounds via a more general fractional operator using generalized Mittag-Leffler function in the kernel, Comput. Model. Eng. Sci., 126 (2021), 359-378. 1

[31] Y. Ren, J. Wang, L. Y. Hu, Multi-valued stochastic differential equations driven by G-Brownian motion and related stochastic control problems, Internat. J. Control, 90 (2017), 1132-1154. 1, 4.2

[32] R. Sakthivel, S. Suganya, S. M. Anthoni, Approximate controllability of fractional stochastic evolution equations, Comput. Math. Appl., 63 (2012), 660-668. 1

[33] G. J. Shen, Y. Ren, Neutral stochastic partial differential equations with delay driven by Rosenblatt process in a Hilbert space, J. Korean Statist Soc., 44 (2015), 123-133. 2

[34] P. Tamilalagan, P. Balasubramaniam, Moment stability via resolvent operators of fractional stochastic differential inclusions driven by fractional Brownian motion, Appl. Math. Comput., 305 (2017), 299-307. 1

[35] C. A. Tudor, Analysis of the Rosenblatt process, ESAIM Probab. Stat., 12 (2008), 230-257. 1

[36] L. W. Wang, Approximate boundary controllability for semilinear delay differential equations, J. Appl. Math., 2011 (2011), 10 pages. 1

[37] S.-S. Zhou, S. Rashid, S. Parveen, A. O. Akdemir, Z. Hammouch, New computations for extended weighted functionals within the Hilfer generalized proportional fractional integral operators, AIMS Math., 6 (2021), 4507-4525. 1 